\title{
Analysis of Transient Thermal Stresses in Annular Fin
}

\author{
Hasan Bas ${ }^{1}$, İbrahim Keles ${ }^{2}$ \\ ${ }^{1}$ Industrial Engineering Department, Ondokuz Mayis University \\ Samsun, Turkey \\ Hasan.bas@omu.edu.tr \\ ${ }^{2}$ Mechanical Engineering Department, Ondokuz Mayis University \\ Samsun, Turkey \\ Ibrahimkeles@omu.edu.tr.
}

\begin{abstract}
The thermal stresses of annular fin with its base subjected of a decayed exponential function of time is examined in this study. The problems are solved by analytically in the Laplace domain and obtained results are converted to real-time space using the modified Durbin's numerical inverse Laplace transform method. Novel approach is using the modified Durbin's numerical inverse Laplace transform method to analysis of transient thermal stresses in annular fin. This method successfully applied the boundary value problem which can be solved in Laplace domain. Different material models from literature are used and temperature and stress distributions are calculated. Validation of the recommended method is done using analytical solutions available in the literature for some special cases, and nearly exact results are obtained.
\end{abstract}

Keywords: Annular fins, transient thermal stress, Laplace transform method, Durbin Laplace transform method

\section{Introduction}

In this study, transient thermal stresses of an annular fin with its base subjected of a decayed exponential function of time are analyzed theoretically in Laplace domain. Thin, one dimensional axisymmetric, isotropic, homogeneous and all thermal properties constant fin is regarded. Laplace transform method is used commonly and partial differential equations are converted to ordinary differential equations using this method. Then these equations can be solved in Laplace domain. Passing Laplace domain to real domain is realized by both numerically and analytically. Obtained solutions in Laplace domain are converted to time domain by using Durbin's inverse Laplace transform method. Temperature and stress distribution throughout the fin thickness are tried to find out. In this novel, the problems that solved by analytical residue method in previous published study are obtained numerically by using Durbin's method [1].

Annular fins are generally used that systems working high temperature like carrying hot fluid in pipes to increase heat transfer from pipes to surrounding fluid [2]. Air cooling internal combustion engines, liquid-gas heat exchangers that used cooling systems and nuclear waste stored tanks are good examples for annular fin [3]. Mokheimer [4] investigated thermal performance of annular fin that have different profile. Fins convection ratio is changed by position. Laor and Kalman [5] calculated efficiency of annular fin assuming heat convection ratio is changed with temperature to determine optimum design parameter of the annular fin. Arauzo et al. [6] determine temperature distribution of annular fin which has hyperbolic profile by using analytic method. Diez et al. [7] research thermal analysis of hyperbolic profile pin fin. Heat convection ratio of fin material is assumed to be constant in previously mentioned studies.

\section{Analysis}

\subsection{Analysis of Thermal Stresses in Annular Fin}

One dimensional, axisymetric annular fin is regarded. This fin has small thickness and constant. The fin material is homogenous, isotropic and all thermal properties are constant. Because of the small fin tip area, heat transfer is neglected so that fin tip is assumed to be isolated. The annular fin structure is shown Fig. 1. 


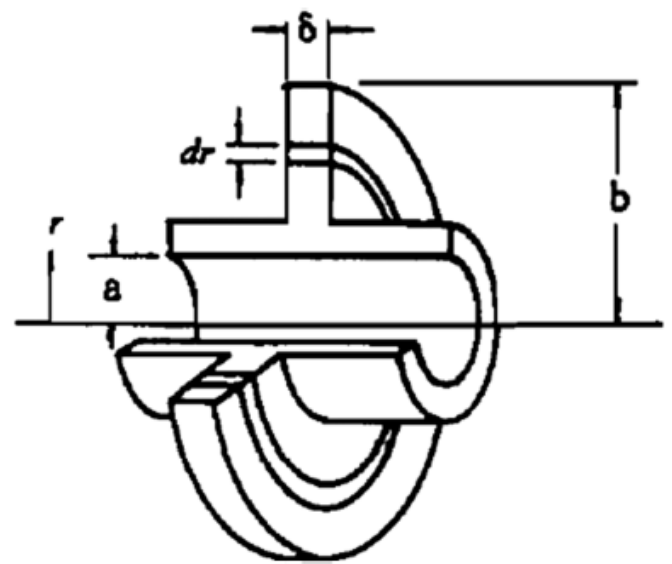

Fig. 1: Annular fin structure.

Dimensionless stress and temperature distribution equations are presented in Equation (1)-(6).

$$
\begin{gathered}
\overline{\sigma_{r}}=-\frac{1}{\zeta^{2}} \int_{1}^{\zeta} \varphi \zeta d \zeta+\frac{1}{\zeta^{2}} \frac{\zeta^{2}-1}{R^{2}-1} \int_{1}^{R} \varphi \zeta d \zeta \\
\overline{\sigma_{\theta}}=-\varphi+\frac{1}{\zeta^{2}} \int_{1}^{\zeta} \varphi \zeta d \zeta+\frac{1}{\zeta^{2}} \frac{\zeta^{2}-1}{R^{2}-1} \int_{1}^{R} \varphi \zeta d \zeta \\
\frac{\partial}{\partial \zeta}\left(\zeta \frac{\partial \varphi}{\partial \zeta}\right)-N^{2} \zeta \varphi=\zeta \frac{\partial \varphi}{\partial \tau}>0, \quad 1<\zeta<R \\
\varphi(\zeta, 0)=0 \quad 1<\zeta<R \\
-\left.\frac{\partial \varphi}{\partial \zeta}\right|_{\zeta=1}=e^{-\omega \tau} t>0 \\
\left.\frac{\partial \varphi}{\partial \zeta}\right|_{\zeta=R}=0 \quad t>0
\end{gathered}
$$

Equations (3)-(6) are initial and boundary value problems.

If we take Laplace transform with respect to time, the system becomes a boundary value problem.

$$
\frac{\partial}{\partial \zeta}\left(\zeta \frac{\partial \bar{\varphi}}{\partial \zeta}\right)-\left(N^{2}+p\right) \zeta \bar{\varphi}=0
$$




$$
\begin{gathered}
\left.\frac{\partial \bar{\varphi}}{\partial \zeta}\right|_{\zeta=1}=\frac{1}{p+\omega} \\
\left.\frac{\partial \bar{\varphi}}{\partial \zeta}\right|_{\zeta=R}=0
\end{gathered}
$$

Where $\mathrm{p}$ is Laplace parameter [1].

Solution of Equations (7) and (8) area presented Equation (10). We can find the all solutions in Bas and Keles's study

$$
\bar{\varphi}=\frac{1}{\sqrt{\zeta}} \frac{\cosh \left[(R-\zeta) \sqrt{N^{2}-p}\right]}{(p+\omega)\left\{(1 / 2) \cosh \left[(R-\zeta) \sqrt{N^{2}-p}\right]+\sqrt{N^{2}-p} \sinh \left[(R-\zeta) \sqrt{N^{2}-p}\right]\right\}}
$$

\subsection{Durbin Inverse Laplace Transform Method}

Numeric inverse Laplace transform method is important to obtain value in time domain. For this purpose, Durbin's Laplace transform method that based on fast Fourier transform is used in this novel. Durbin inverse Laplace transform equations are summarized in Equations (11)-(14):

$$
\begin{gathered}
\tilde{f}\left(\zeta, \xi_{j}\right)=\frac{2 \operatorname{Exp}[a j \Delta t]}{T}\left[-\frac{1}{2} \operatorname{Re}\{\tilde{f}(\zeta, a)\}+\operatorname{Re}\left\{\sum_{k=0}^{N-1}(A(\zeta, k)+i B(\zeta, k)) W^{j k}\right\}\right], \\
j=0,1,2, \ldots, N-1 \\
A\left(\zeta, \xi_{j}\right)=\sum_{m=1}^{M} \operatorname{Re}\left\{x\left\{\tilde{f}\left(\zeta, a+i(k+m N) \frac{2 \pi}{T}\right)\right\}\right\} \\
B\left(\zeta, \zeta_{j}\right)=\sum_{m=1}^{M} \operatorname{Im}\left\{\tilde{f}\left(\zeta, a+i(k+m N) \frac{2 \pi}{T}\right)\right\} \\
W=\operatorname{Exp}\left[i \frac{2 \pi}{N}\right], \quad \Delta t=\frac{T}{N}
\end{gathered}
$$

$\mathrm{z}_{\mathrm{k}}=\mathrm{a}+\mathrm{ik} \frac{2 \pi}{\mathrm{T}}$ and $\mathrm{N}=\frac{\mathrm{T}}{\Delta \mathrm{t}}, \mathrm{k}$ th degree Laplace transform parameter, $T$ is solution time and $\Delta t$ is time increment. Choosing $a$ constant value in inverse Laplace transform is explained by Durbin [8]. According to Durbin's study we need to set $a T$ value between 5 and 10 to getting better result. Because of that $a T$ value is set 7.5 in this study. Terminally the solutions are multiplied with Lanczos $L_{k}$ factor and best solutions are obtained [9]. 


$$
L_{k}=\sin \left(\frac{k \pi}{N}\right) /\left(\frac{k \pi}{N}\right)
$$

\section{Results}

Obtained numeric solutions are compared with Shang-Shen Wu's analytical solutions [10]. Numerical solutions and comparing are shown Figures 2-7. Whereas obtained solutions in this study are shown with symbols, referents solutions are shown by lines in all figures.

Fig. 2 exhibits the dimensionless hoop stress distribution $\overline{\sigma_{\theta}}$ of the fin versus the dimensionless radius $\zeta$ for different value of $\mathrm{N}$ at $\tau=0.5, \omega=0.5$ and $\mathrm{R}=2$. Dimensionless hoop stress decreases at until the value of dimensionless radius parameter $\zeta$ is 1.3-1.4. There are compressive stresses in this region. After this region there are tensile stresses. Before this region, increasing $\mathrm{N}$ parameter causes increasing compressive stresses. However after this region increasing $\mathrm{N}$ parameter causes increasing tensile stresses.

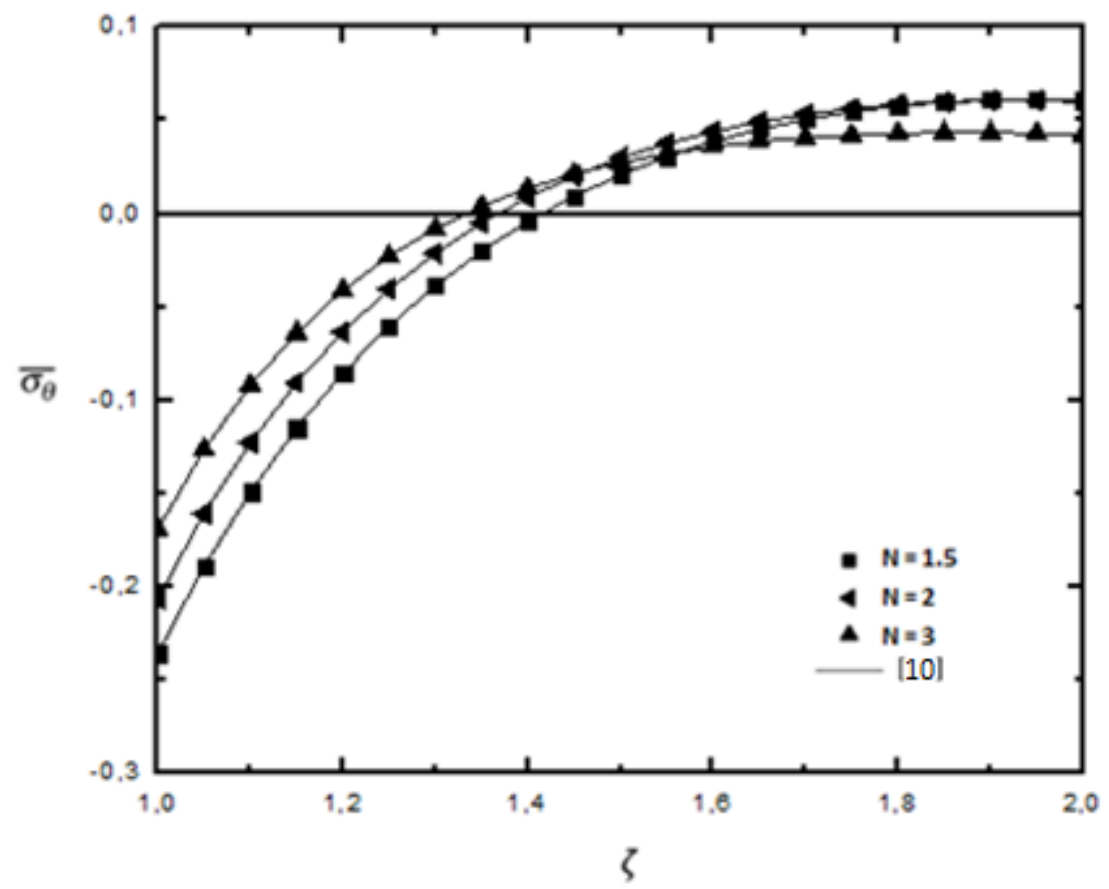

Fig. 2: Dimensionless hoop stress distribution for different $N$ values at $\tau=0.5, \omega=0.5$ and $R=2$.

The dimensionless temperature distribution $\varphi$ of the fin versus the dimensionless time parameter $\tau$ for different value of $\mathrm{N}$ at $\zeta=1, \omega=0.5$ and $\mathrm{N}=2$ is shown in Figure 3. All dimensionless temperature value for different $\mathrm{R}$ is equal till the dimensionless time parameter value $\tau$ is 0.15 . Dimensionless temperature decreases after this value. Moreover dimensionless temperature increase till the $\tau=0.3$, after this point temperature decreases. 


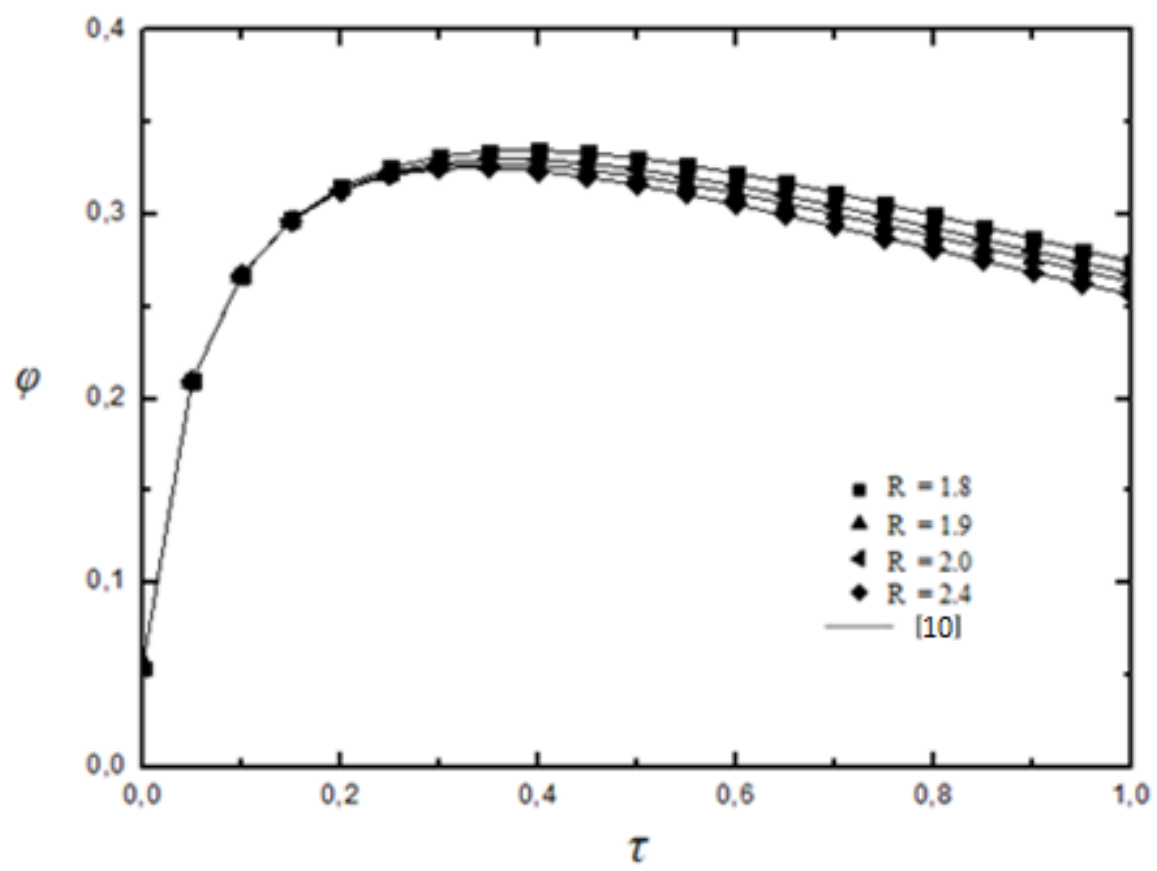

Fig. 3: Dimensionless temperature distribution for different $R$ values at $\zeta=1, \omega=0.5$ and $N=2$.

The dimensionless temperature distribution $\varphi$ versus the dimensionless time parameter $\tau$ for different value of $R$ at $\zeta=R, \omega=0.5$ and $N=2$ is viewed in Figure 4. We can infer from the figure that increasing $R$ values causes to decreasing temperature values. Temperature value is generally highest where the dimensionless time parameter $\tau$ is 0.7 and after this point temperature decreases.

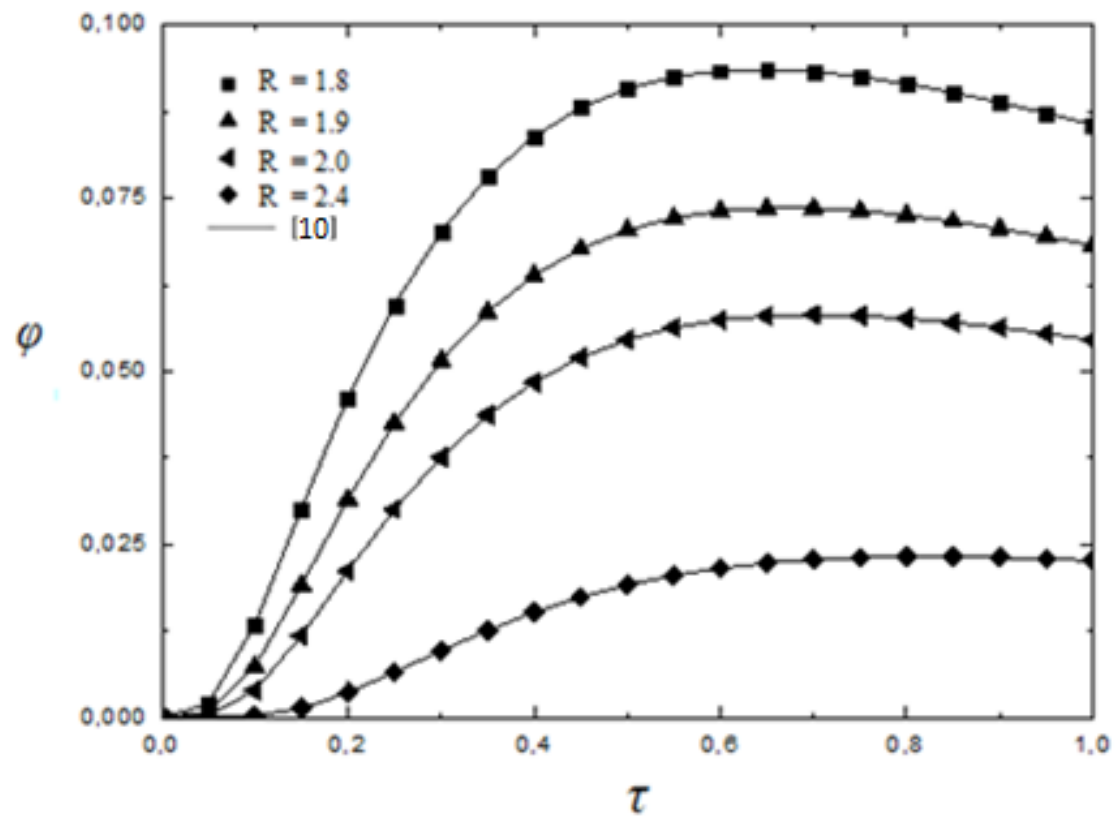

Fig.4: The dimensionless temperature distribution for different $R$ value at $\zeta=R, \omega=0.5$ and $N=2$.

The dimensionless temperature distribution $\varphi$ of the fin versus the dimensionless time parameter $\tau$ for different $N$ value at $\zeta=1, \omega=0.5$ and $R=2$ is shown in Figure 5. We can infer from the figure that increasing $N$ values causes to decreasing temperature values. 


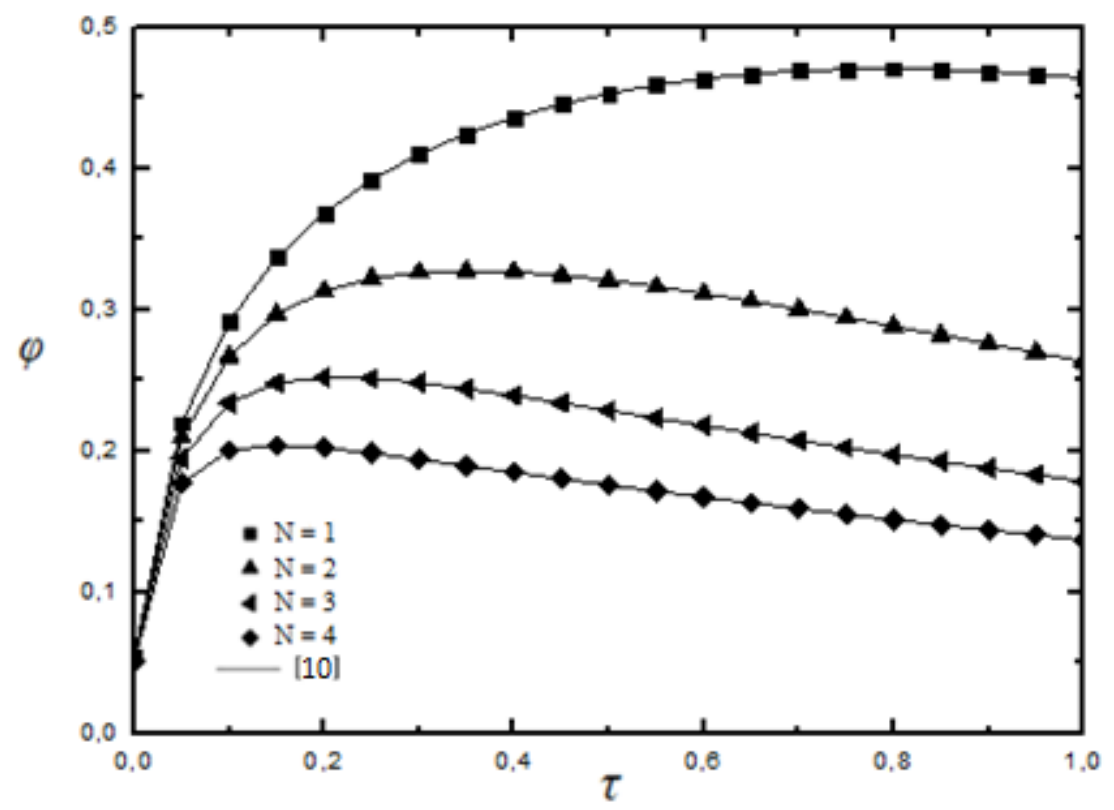

Fig. 5: The dimensionless temperature distribution for different $\mathrm{N}$ value at $\zeta=1, \omega=0.5$ and $R=2$.

The dimensionless temperature distribution $\varphi$ of the fin versus the dimensionless time parameter $\tau$ for different $\mathrm{N}$ value at $\zeta=\mathrm{R}, \omega=0.5$ and $\mathrm{R}=2$ is shown in Figure 6. Increasing $\mathrm{N}$ values causes to decreasing temperature values.

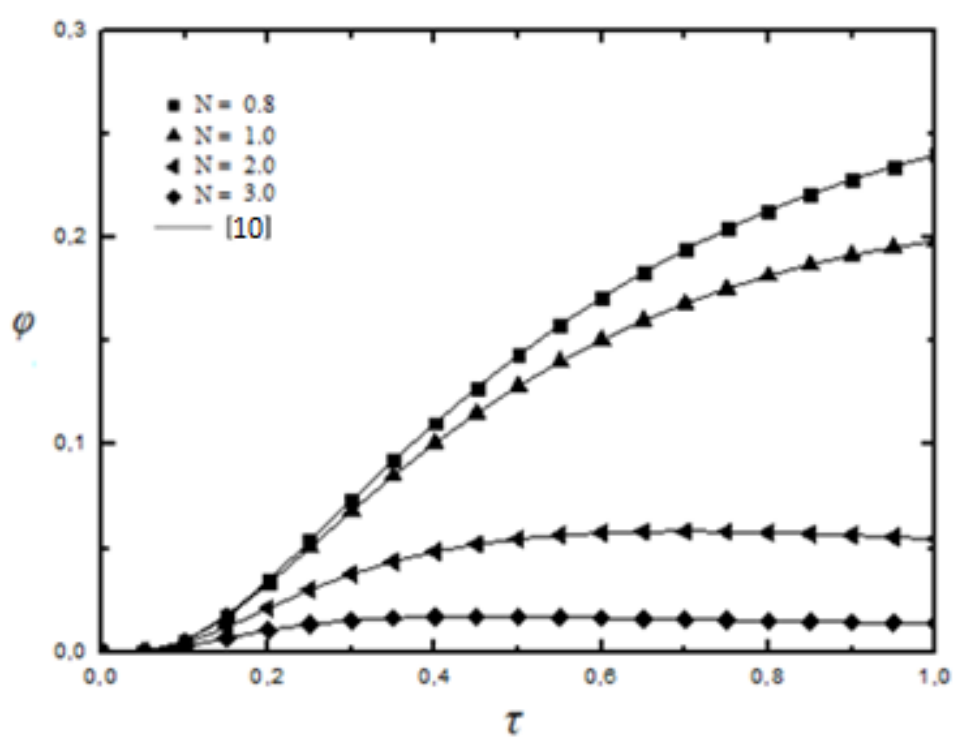

Fig. 6: The dimensionless temperature distribution for different $N$ values at $\zeta=R, \omega=0.5$ and $R=2$.

The dimensionless temperature distribution $\varphi$ of the fin versus the dimensionless time parameter $\tau$ for different $\mathrm{N}$ values at $\zeta=(1+\mathrm{R}) / 2, \omega=0.5$ and $\mathrm{N}=2$ is shown in Figure 7 . We can infer from the figure that increasing $\mathrm{R}$ values causes to decreasing temperature values. Temperature value is generally highest where the dimensionless time parameter $\tau$ is 0.6 and after this point temperature decreases. 


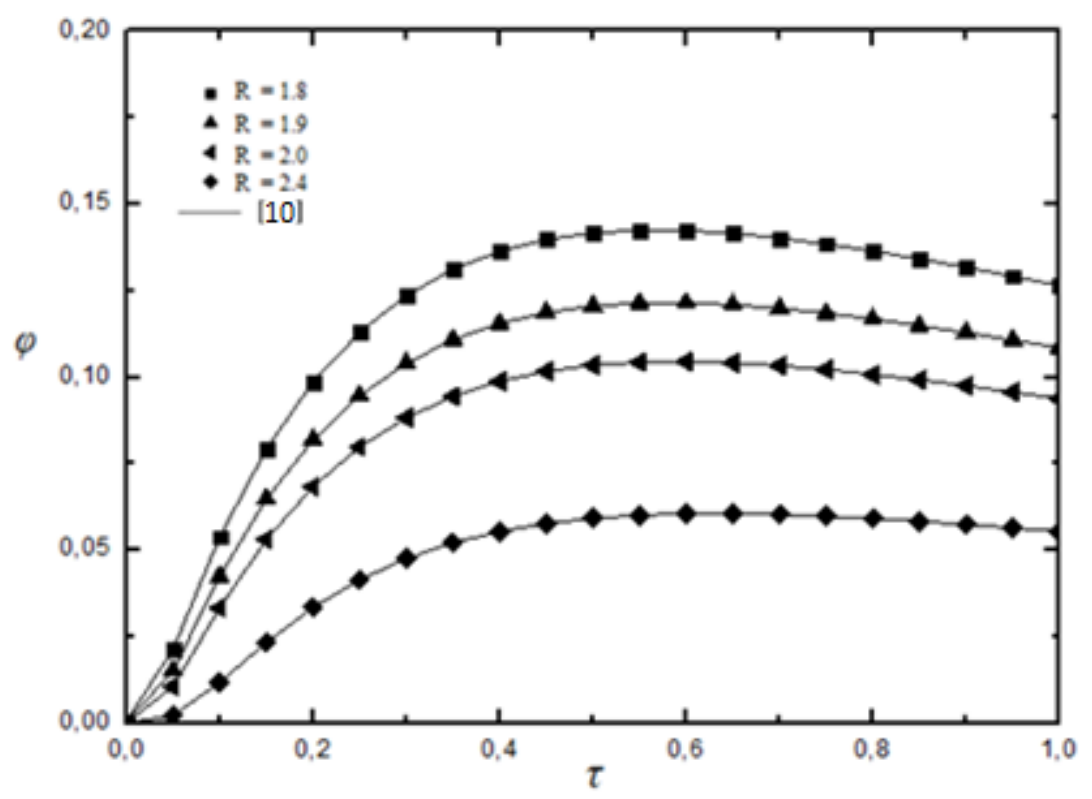

Fig. 7: The dimensionless temperature distribution for different $R$ value at $\zeta=(1+\mathrm{R}) / 2, \omega=0.5$ and $\mathrm{N}=2$.

\section{Conclusion}

Temperature and stress distributions are shown in graphics. Numerical results that obtain for all situations are presented. Additionally obtained solutions are coincided with the analytical Shang-Shen Wu [10] solutions.

We can summarize the results like followings from this study:

1.Temperature of the fin increases in progress of time. However temperature begins to decrease after the approaching highest temperature and finally temperature reaches steady state. Whereas all stresses are compressive between the midpoint and inner radius, all stresses are tensile in the other regions.

2. In spite of the increasing of the dimensionless outer radius $R$ and the dimensionless inner radius $\zeta$, temperature values decrease. If we examine the dimensionless hoop stress $\overline{\sigma_{\theta}}$, we can see that increasing the dimensionless outer radius $R$ causes high stress in compressive region but it causes low stress in tensile region.

3.Increasing of the dimensionless $N$ parameter causes decreasing temperature of fin. Because higher $N$ value means that higher heat transfer ratio, so that more heat transfer from fin surface is occurred.

4.In this study, applying Laplace transform to time dependent differential equation and then applying the modified Durbin's inverse Laplace transform method, considerable and effective solutions are found with comparing the analytical solution in literature.

\section{Nomenclature}

$a$ : Outer radius of the fin $(m)$

$\boldsymbol{b}$ : Inner radius of the fin $(m)$

$\zeta$ : Dimensionless radius $(r / a)$

$\boldsymbol{R}$ : Dimensionless outer radius $(r / b)$

$\boldsymbol{\varphi}$ : Dimensionless temperature of the fin

$\overline{\sigma_{r}} \quad:$ Dimensionless radial stress

$\overline{\sigma_{\theta}}:$ Dimensionless tangential stress

$\boldsymbol{\delta}:$ Thickness of the fin

$\boldsymbol{N}$ : Dimensionless parameter $\left(N^{2}=2 h a^{2} / k \delta\right)$

$\boldsymbol{t}:$ : Time

$\boldsymbol{L}_{\boldsymbol{k}}$ : Lanczos factor

$\tau$ : Dimensionless time 
$\boldsymbol{h}:$ Heat transfer coefficient

$\boldsymbol{k} \quad$ : Thermal conductivity of material of the fin

$\boldsymbol{\rho} \quad$ : Density $\left(\mathrm{kg} / \mathrm{m}^{3}\right)$

c : Specific heat of material of the fin $(\mathrm{J} / \mathrm{gr} . \mathrm{K})$

\section{References}

[1] H. Bas and İ. Keles, "Novel approach to transient thermal stress in an annular fin," Journal of Thermophysics and Heat Transfer, vol. 29, no. 4, pp. 705-710, 2015.

[2] A. D. Kraus, A. Aziz, J. R. Welty, Extended Surface Heat Transfer, McGraw-Hill, New York, 2001.

[3] A. Campo, J. Cui, "Temperature/heat analysis of annular fins of hyperbolic profile relying on the simple theory for straight fins of uniform profile," Journal of Heat Transfer, vol. 130, no. 054501, pp. 1-4, 2008.

[4] E. M. A. Mokheimer, "Performance of annular fins with different profiles subject to variable heat transfer coefficient," International Journal of Heat and Mass Transfer, vol. 45, no. 17, pp. 3631-3642, 2008.

[5] K. Laor, H. Kalman, "Performance and optimum dimensions of different cooling fins with a temperature dependent heat transfer coefficient," International Journal of Heat and Mass Transfer, vol. 39, no. 9, pp. 1993-2003, 1996.

[6] I. Arauzo, A Campo, C. Cortes, "Quick estimate of the heat transfer characteristics of annular fins of hyperbolic profile with the power series method," Applied Thermal Engineering, vol. 25, no. 4, pp. 623-634, 2005.

[7] L. I. Diez, A. Campo, C. Cortes, "Quick design of truncated pin fins of hyperbolic profile for heat-sink applications by using shortened power series," Applied Thermal Engineering, vol. 29, no. 5-6, pp. 815-821, 2009.

[8] F. Durbin, "Numerical Inversion of Laplace Transform: An Efficient Improvement ot Dubner and Abate's Method," Computer Journal, vol. 17, no. 14, pp. 371-376, 1974.

[9] F. F. Calim, Dynamics “Analysis of Beams on Viscoelastic Foundation," European Journal of Mechanics, A: Solids, vol. 28, no. 3, pp. 469-476, 2009.

[10] S. S. Wu, “Analysis on Transient Thermal Stress in an Annular Fin,” Journal of Thermal Stresses, vol. 20, no. 6, pp. 591-615, 1997. 